\title{
Timing of Arteriovenous Fistula Placement and Medicare Costs during Dialysis Initiation
}

\author{
Craig A. Solid ${ }^{a}$ Caroline Carlin ${ }^{b, c}$ \\ aUnited States Renal Data System, Minneapolis Medical Research Foundation, Minneapolis, Minn., \\ ${ }^{b}$ Medica Research Institute, Minnetonka, Minn., and ' Department of Applied Economics, University of Minnesota, \\ St. Paul, Minn., USA
}

\section{Key Words}

Hemodialysis • Endogenous selection · Selection bias ·

Vascular access

\begin{abstract}
Background/Aims: Arteriovenous fistulas (AVFs) appear to be clinically superior to catheters as vascular access for maintenance hemodialysis, but higher insertion costs and high disease burden and mortality obscure the issue of whether AVF placement before hemodialysis initiation represents a net cost savings. We aimed to investigate Medicare costs for patients beginning maintenance hemodialysis, as related to timing of AVF placement. Methods: Data were from Medicare claims for incident hemodialysis patients aged $\geq 67$ years in 2006. The study period extended from 2 years before to 1 year after dialysis initiation. Patients identified as having AVFs were categorized by timing of placement (mature AVF at dialysis initiation, maturing AVF at initiation, postinitiation AVF placement). Because timing may be influenced by factors that also influence overall costs, the model accounted for this nonrandom treatment assignment. An ordered probit extension of the classic Heckman correction was employed after identifying an appropriate instrumental variable. A cohort with Medicare coverage before and after dialysis initiation was identified, and Medicare claims were
\end{abstract}

used to identify comorbid conditions and treatment costs. Results: Principal findings are that earlier AVF placement leads to lower costs, with the potential for about USD 500 million in savings. Additionally, the effect of nonrandom treatment assignment is real and significant. In our data, the impact of AVF placement timing was understated when treatment selection was ignored. Conclusions: For appropriate AVF candidates, having a mature AVF in place at the time of dialysis initiation appears to confer cost savings.

Copyright $\odot 2012$ S. Karger AG, Basel

\section{Introduction}

Prevalence of end-stage renal disease (ESRD) requiring renal replacement therapy with dialysis or kidney transplant has grown substantially in the last half century. More than 570,000 ESRD patients currently reside in the USA, a number projected to reach almost 800,000 by $2020[1,2]$. Kidney transplants have become common; however, due to organ shortages and the complex disease burden, the majority (90\%) of new ESRD patients undergo clinic-provided hemodialysis as ESRD treatment [2]. Catheters for hemodialysis vascular access are inexpensive and quick to insert, but prone to infections and complications such as thrombosis. Long-

\section{KARGER}

Fax +4161306 1234

E-Mail karger@karger.ch

www.karger.com
(C) 2012 S. Karger AG, Basel

0250-8095/12/0356-0498\$38.00/0

Accessible online at:

www.karger.com/ajn
Craig A. Solid, $\mathrm{PhD}$

United States Renal Data System

Minneapolis Medical Research Foundation

914 South Eighth Street, Suite S-406, Minneapolis, MN 55404 (USA)

Tel. +1 612337 7361, E-Mail csolid@ usrds.org 
term use can reduce the likelihood that a permanent access can eventually be placed because catheters can cause vein stenosis $[3,4]$.

Because arteriovenous fistulas (AVFs) are surgically created, they are more expensive than catheters and require time to mature before they can be used. However, they have been shown to be at lower risk for infections and complications [5], and are associated with lower rates of morbidity and mortality [6-19]. Patients using AVFs tend to have better clinical markers and require lower doses of typical drug therapies such as intravenous iron and erythropoietin [20-22]. Clinical guidelines established by the National Kidney Foundation suggest placing an AVF before the patient develops ESRD so it is mature by the time regular dialysis begins [23]. However, Medicare does not cover the first 90 days of ESRD treatment for patients not already eligible for Medicare. A motivation for this study was to investigate whether Medicare coverage of AVF placement before dialysis initiation would result in a net savings.

Not all patients are candidates for AVFs; those who are frail or have small veins may not be able to maintain such an access [24]. Therefore, when comparing outcomes of patients using catheters and patients using AVFs, it is important to note that type of vascular access is not randomly assigned. Several factors (including unmeasured factors) may influence which access a patient uses, and those same factors may affect health-related outcomes. This nonrandom assignment of treatment group (catheter vs. AVF) is often referred to as 'selection bias' since it can lead to statistically biased parameter estimates if it is not accounted for. Previous studies attempting to assess the cost benefit or cost utility of the type of vascular access used for hemodialysis [25, 26] and the National Institutes of Health/National Institute of Diabetes and Digestive and Kidney Diseases [27] have typically ignored the issue of nonrandom treatment assignment.

While AVFs appear to be clinically superior to catheters, higher insertion costs and the high disease burden and mortality of patients before and immediately after ESRD onset [28] obscure the issue of whether AVF placement before hemodialysis initiation represents a net cost savings. Additionally, the selection issue inherent in vascular access type makes comparing AVFs and catheters challenging. This study aimed to assess whether having a mature AVF at the time of dialysis initiation represents a net cost savings compared with having an AVF that is still maturing at initiation or is placed after initiation, while addressing the selection issue.

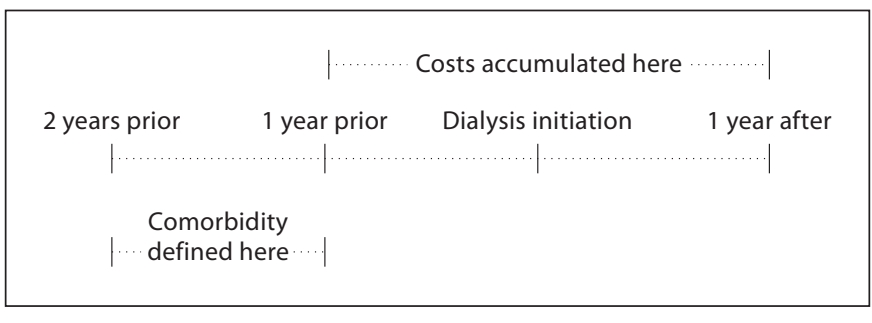

Fig. 1. Timing of Medicare claims used to identify comorbidity and to calculate costs.

\section{Methods}

\section{Study Design and Data Sources}

The United States Renal Data System (USRDS) serves as the registry and data warehouse for all ESRD patients in the USA. Data available from the USRDS include de-identified patient information and health encounter and cost information from Medicare claims (treatment of ESRD has been covered by Medicare since 1973). To use these data, we identified a cohort with Medicare coverage before and after dialysis initiation and used Medicare claims to identify health encounters, including the timing of AVF placement. The study period extended from 2 years before through 1 year after dialysis initiation; patients were censored at death or change in ESRD treatment (primarily kidney transplant). The cohort was limited to patients who had an AVF placed during the study period in an attempt to exclude patients who were not candidates for AVFs. Thus, this study evaluated the impact of timing of AVF placement, not the impact of AVF use. Demographic and patient information was obtained from the USRDS database, and disease burden was established from Medicare claims before dialysis initiation. Cost information was gathered from Medicare claims and represents the total cost to Medicare from 1 year before through 1 year after dialysis initiation. Figure 1 details the timing of claims used to identify comorbidity and calculate costs.

Since the claims reflect all health encounters paid for by Medicare, total costs include the cost of AVF placement, hospitalizations, infectious events, access-specific procedures (thromboses, revisions, replacements, etc.), and any other events that result in a Medicare claim. Patients who died or changed ESRD treatment within the first year after dialysis initiation were included, but their costs were censored at date of death or treatment change. County-level information on the average Medicare reimbursement amount per beneficiary was obtained from the Dartmouth Atlas to allow adjustment for geographic differences in Medicare reimbursement and geographically based differences in practice patterns.

\section{Cohort and Treatment Group Assignment}

We identified 46,551 new hemodialysis patients in 2006 who were aged $\geq 67$ years at dialysis initiation (to ensure 2 years of Medicare coverage before dialysis initiation). A total of 16,863 patients were excluded because of health maintenance organization coverage or insurance payer status; of the remaining patients, 12,336 were identified as having had an AVF placed during the 
Table 1. Treatment group characteristics

\begin{tabular}{|c|c|c|c|}
\hline & \multicolumn{3}{|c|}{ AVF treatment group } \\
\hline & mature & maturing & postinitiation \\
\hline Total, n & 3,046 & 2,381 & 6,909 \\
\hline \multicolumn{4}{|l|}{ Age, years } \\
\hline Mean & 76.2 & 76.4 & 76.5 \\
\hline Median & 76 & 76 & 76 \\
\hline $65-74$ & $1,291(42.4)$ & $999(42.0)$ & $2,819(40.8)$ \\
\hline $75-84$ & $1,443(47.4)$ & $1,135(47.7)$ & $3,338(48.3)$ \\
\hline$\geq 85$ & $312(10.2)$ & $247(10.4)$ & $752(10.9)$ \\
\hline \multicolumn{4}{|l|}{ Race } \\
\hline White & $2,485(81.6)$ & $1,864(78.3)$ & $5,421(78.5)$ \\
\hline African-American & $424(13.9)$ & $436(18.3)$ & $1,260(18.2)$ \\
\hline Other & $137(4.5)$ & $81(3.4)$ & $228(3.3)$ \\
\hline \multicolumn{4}{|l|}{ Sex } \\
\hline Men & $1,951(64.1)$ & $1,321(55.5)$ & $3,808(55.1)$ \\
\hline Women & $1,095(35.9)$ & $1,060(44.5)$ & $3,101(44.9)$ \\
\hline \multicolumn{4}{|l|}{ Comorbid conditions } \\
\hline ASHD & $1,236(40.6)$ & $1,102(46.3)$ & $2,828(40.9)$ \\
\hline Congestive heart failure & $914(30.0)$ & $835(35.1)$ & $2,329(33.7)$ \\
\hline Cerebrovascular accident & $385(12.6)$ & $344(14.4)$ & $933(13.5)$ \\
\hline PVD & $740(24.3)$ & $594(24.9)$ & $1,579(22.9)$ \\
\hline Other cardiac disease & $680(22.3)$ & $558(23.4)$ & $1,566(22.7)$ \\
\hline COPD & $501(16.4)$ & $441(18.5)$ & $1,329(19.2)$ \\
\hline GI disease & $183(6.0)$ & $157(6.6)$ & $504(7.3)$ \\
\hline Liver disease & $38(1.2)$ & $22(0.9)$ & $115(1.7)$ \\
\hline Dysrhythmia & $733(24.1)$ & $637(26.8)$ & $1,805(26.1)$ \\
\hline Cancer & $401(13.2)$ & $299(12.6)$ & $814(11.8)$ \\
\hline Diabetes & $1,658(54.4)$ & $1,487(62.5)$ & $3,821(55.3)$ \\
\hline Anemia & $2,140(70.3)$ & $1,408(59.1)$ & $3,246(47.0)$ \\
\hline CKD & $2,663(87.4)$ & $1,775(74.5)$ & $3,767(54.5)$ \\
\hline Hypertension & $2,827(92.8)$ & $2,130(89.5)$ & $5,744(83.1)$ \\
\hline Cognitive impairment & $43(1.4)$ & $29(1.2)$ & $105(1.5)$ \\
\hline Depression & $134(4.4)$ & $117(4.9)$ & $348(5.0)$ \\
\hline Wheelchair use & $443(14.5)$ & $495(20.8)$ & $1,689(24.4)$ \\
\hline Nephrology days ${ }^{1}$ (mean) & 546.3 & 459.6 & 336.4 \\
\hline Nephrology days ${ }^{1}$ (median) & 637 & 555 & 315 \\
\hline Died within 1 year of ESRD & $518(17.0)$ & $612(25.7)$ & $1,697(24.6)$ \\
\hline Kidney transplant within 1 year & $41(1.3)$ & $14(0.6)$ & $13(0.2)$ \\
\hline Mean days to death (those who died) & 174.4 & 158.5 & 206.96 \\
\hline Mean cost, USD & 103,869 & 119,022 & 127,677 \\
\hline
\end{tabular}

Unless otherwise indicated, values are $\mathrm{n}(\%)$. ASHD $=$ Atherosclerotic heart disease; AVF = arteriovenous fistula; $\mathrm{CKD}=$ chronic kidney disease; $\mathrm{COPD}=$ chronic obstructive pulmonary disease; $\mathrm{GI}=$ gastrointestinal; $\mathrm{PVD}=$ peripheral vascular disease.

${ }^{1}$ Number of days between first Medicare claim for a nephrologist visit and dialysis initiation.

study period. These patients were categorized into three treatment groups based on the timing of AVF placement: 3,046 patients had a mature AVF at dialysis initiation, 2,381 had a maturing AVF at dialysis initiation, and 6,909 had an AVF placed after dialysis initiation. Comorbid conditions were identified using International Classification of Diseases, Ninth Revision, Clinical Modification diagnosis codes (Appendix) in Medicare claims 1324 months before dialysis initiation.

\section{Comparison of Treatment Groups}

A comparison of characteristics across the three treatment groups (table 1) indicated similar age distributions, but patients with mature AVFs were more likely to be male and of white race. Prevalence of comorbid conditions was relatively similar, but patients with maturing AVFs were more likely to have cardiovascular disease, diabetes mellitus, and atherosclerotic heart disease, all conditions relating to or affecting vascular health. Notably, prev- 
alence of chronic kidney disease (CKD), anemia, and hypertension (all associated with kidney function and likely present to some degree in almost all patients in the cohort) clearly differed across treatment groups. Prevalence of CKD was highest in patients with mature AVFs, at $87 \%$ compared with $75 \%$ for patients with maturing AVFs and 55\% for patients with AVFs placed after dialysis initiation. Patterns of decreasing prevalence across the treatment groups also occurred for anemia and hypertension (respectively, anemia, 70\%, 59\%, 47\%; hypertension, 93\%, 90\%, 83\%). One hypothesis is that higher demonstrated prevalence of these diseases represents earlier recognition of kidney disease, not true prevalence; earlier recognition could influence whether an AVF is placed before dialysis initiation. Because of this concern, these variables were omitted from the selection model and from the cost models. Because the study measured total costs and not rate per time at risk, it is constructive to consider how mortality affects this cost variable. Cumulative 12-month mortality (beginning at dialysis initiation) was similar in the maturing AVF and postinitiation AVF groups, at about 25\% 1 year after dialysis initiation (table 1). This is true despite the slight survival bias in the postinitiation AVF group, whose members had to survive until AVF placement to be included in that group. In comparison, cumulative mortality in the mature AVF group was $17 \%$ at 1 year, reflecting both better outcomes from AVF use and better overall health status. As it relates to this study, however, a lower mortality rate would increase costs since medical expenses for living patients are regular and large due to ongoing dialysis. A more formal treatment of mortality differences is available elsewhere (C.A. Solid and C.S. Carlin, unpubl. data, manuscript submitted for publication, February 2012).

\section{Statistical Analysis}

Claims data typically include only billing information, omitting variables such as disease severity or patient behavior. When these data sources are used to model an outcome by treatment group, these omitted variables can produce bias in the estimation of model parameters. Specifically, the unmeasured characteristics may influence some patients to self-select or to be selected into a particular treatment group, while also affecting the outcome of interest such as total cost of care. For example, some patient behaviors could accelerate kidney function decline (allowing less time for an AVF to mature) and increase the likelihood of hospitalization (increasing total costs). This dual association of unmeasured factors is at the heart of the statistical bias that can result from the nonrandom treatment assignment, and would affect parameter estimates produced by a cost model that ignores the selection issue.

One method for addressing this nonrandom treatment assignment is an ordered probit extension of the Heckman correction [29, 30]. The ordered probit requires a natural ordering of the dependent variable, which in this case represents the timing of AVF placement, and in turn membership in one of the three groups: (1) mature AVF at dialysis initiation, (2) maturing AVF at initiation, or (3) AVF placement delayed until after initiation. As applied here, costs are modeled as a log-linear regression dependent on AVF status and other patient characteristics. The error terms of the ordered probit selection model and log-linear cost model are assumed to be bivariate normal and correlated. The ordered probit selection model produces an estimate that is an extension of the inverse Mills' ratio first developed by Heckman [29], which is in- cluded in the cost model to account for the correlation. Details of this ordered probit methodology, and of the 'oheckman' Stata command that employs it, can be found in Chiburis and Lokshin [30].

This ordered probit Heckman correction requires an instrumental variable (IV) to identify the group selection. Success of this method depends on availability of a good instrument, one directly related to group selection but not to overall costs. The IV chosen for this analysis was timing of referral to a nephrologist before ESRD onset. This variable ('NephDays') was calculated by counting the days between the date of the first Medicare Part B claim on which the indicated physician specialty was nephrology and the date of dialysis initiation. Clinically, one would expect that the earlier patients are seen by a nephrologist, the earlier they are likely to have an AVF placed. We found that the first nephrology claim appears, on average, 17.9 months before initiation for the mature AVF group, 15.1 months before for the maturing AVF group, and 11.0 months before for the postinitiation AVF group. One would not expect early nephrologist referral to be directly related to costs of dialysis treatment because most costs associated with ESRD are incurred after dialysis initiation, at which point nearly all patients are seeing a nephrologist. For patients with no nephrologist claim before initiation, the variable is set equal to zero. There is no formal test for the appropriateness of a single IV. However, the descriptive data in table 1 show an association between early referral to a nephrologist and early AVF placement. Graphical analysis (available from the corresponding author) showed no link between length of relationship with a nephrologist and total cost of care, in support of the variable's role as an IV.

\section{Results}

\section{Initial Cost Comparisons}

On average, overall total costs were lowest for the mature AVF group and progressively higher for the maturing AVF and postinitiation AVF groups. Mean costs during the study period for patients in these groups were USD 103,869, USD 119,022, and USD 127,677, respectively (table 1). As noted, mean cost was lowest for the mature AVF group, even with favorable mortality. We believed that modeling total cost, rather than a measure such as cost per month, was important to facilitate an assessment of the cost effectiveness of early AVF placement because of the distinct pattern of costs clustered around dialysis initiation.

It is not surprising that total Medicare costs are skewed and require data transformation before they can be modeled as an approximately normally distributed dependent variable. Methods detailed in previous studies [31, 32] were used to determine that log-transformation was appropriate for these data. After model fitting, predicted values for $\log$ (cost) were retransformed using appropriate smearing methods [33] while considering possible heteroskedasticity. 
Table 2. Results of ordered probit selection model ${ }^{1}$

\begin{tabular}{|c|c|c|}
\hline & Coefficient & $\mathrm{p}$ \\
\hline \multicolumn{3}{|l|}{ Nephrology days decile ${ }^{2}$} \\
\hline 2 & 0.8365 & 0.000 \\
\hline 3 & 0.3392 & 0.000 \\
\hline 4 & -0.2824 & 0.000 \\
\hline 5 & -0.491 & 0.000 \\
\hline 6 & -0.6368 & 0.000 \\
\hline 7 & -0.6572 & 0.000 \\
\hline 8 & -0.7846 & 0.000 \\
\hline 9 & -0.8014 & 0.000 \\
\hline 10 & -0.8038 & 0.000 \\
\hline Age & 0.0034 & 0.077 \\
\hline Female sex & 0.1259 & 0.000 \\
\hline African-American race & 0.1146 & 0.000 \\
\hline Other race & -0.1021 & 0.086 \\
\hline ASHD & 0.0401 & 0.127 \\
\hline Congestive heart failure & 0.1066 & 0.000 \\
\hline Cerebrovascular accident & 0.0327 & 0.333 \\
\hline PVD & -0.0358 & 0.195 \\
\hline Other cardiac disease & -0.0017 & 0.954 \\
\hline COPD & 0.052 & 0.093 \\
\hline GI disease & 0.0654 & 0.161 \\
\hline Liver disease & 0.175 & 0.082 \\
\hline Dysrhythmia & 0.0092 & 0.749 \\
\hline Cancer & -0.0062 & 0.855 \\
\hline Diabetes & -0.0114 & 0.634 \\
\hline Wheelchair uses & 0.2448 & 0.000 \\
\hline $\log$ (total hospital days) & 0.1105 & 0.000 \\
\hline At least 1 hospitalization & -0.1992 & 0.000 \\
\hline \multicolumn{3}{|c|}{ Geographic decile of average reimbursement ${ }^{3}$} \\
\hline 2 & 0.0536 & 0.414 \\
\hline 3 & 0.0965 & 0.149 \\
\hline 4 & 0.1491 & 0.019 \\
\hline 5 & 0.1533 & 0.018 \\
\hline 6 & 0.1739 & 0.006 \\
\hline 7 & 0.1565 & 0.013 \\
\hline 8 & 0.1654 & 0.007 \\
\hline 9 & 0.1821 & 0.003 \\
\hline 10 & 0.1191 & 0.045 \\
\hline Cutoff $1^{4}$ & -0.524 & 0.001 \\
\hline Cutoff $2^{4}$ & 0.0756 & 0.639 \\
\hline LR test $\left(\chi^{2}\right)$ & $2,042.39$ & 0.000 \\
\hline Pseudo $\mathrm{R}^{2}$ & 0.0843 & \\
\hline
\end{tabular}

Reference categories are nephrology days decile 1, male sex, white race, absence of disease, and geographic decile 1 . ASHD = Atherosclerotic heart disease; COPD = chronic obstructive pulmonary disease; $\mathrm{GI}=$ gastrointestinal; $\mathrm{LR}=$ likelihood ratio; PVD = peripheral vascular disease

${ }^{1}$ Ordinal values: $0=$ mature AVF at initiation; $1=$ maturing AVF at initiation; $2=$ AVF placed after initiation. ${ }^{2}$ Number of days between first Medicare claim for a nephrologist visit and dialysis initiation. ${ }^{3}$ County-level deciles based on Dartmouth Atlas average reimbursement per Medicare beneficiary. ${ }^{4}$ Cutoff 1 and cutoff 2 refer to the boundaries on the value of the latent probit variable that define the observed ordinal value.
If the issue of nonrandom treatment assignment is ignored and ordinary least squares regression is applied to $\log (\operatorname{cost})$, statistically significant factors include age, sex, race, and presence of several comorbid conditions, including atherosclerotic heart disease, cerebrovascular accident (stroke), peripheral vascular disease, dysrhythmia, cancer, and diabetes (data not shown). Claims relating to wheelchair use (a frailty measure) and the adjustment for regional differences in average Medicare reimbursement per patient are also significant. However, the parameter estimates for these factors were likely statistically biased due to nonrandom treatment assignment, and for this reason we pursued the selection-corrected cost model (summary cost results are given below).

\section{Selection and Adjusted Cost Models}

As discussed, we used timing of first nephrologist claim (number of days before dialysis initiation, or 'NephDays') as an instrument for treatment group. This variable is significantly nonnormal and includes differing amounts of values near and equal to zero. For this reason, the two-step method of the 'oheckman' command, which involves estimating the selection model and cost model sequentially instead of simultaneously and is more robust to nonnormality, was used to fit the model [30]. In addition, several parameterizations of this variable were tested; categorizing the days of nephrology care by deciles was found to produce the best overall model fit.

The significance of 'NephDays' in predicting the timing of AVF placement in a traditional ordered probit model is shown in table 2. Demographics (age, sex, race, geography) were highly significant, as were many of the comorbidity indicators (atherosclerotic heart disease, congestive heart failure, diabetes, gastrointestinal disease, liver disease) and the frailty measures (wheelchair claims, $\log$ [hospital days], hospitalization indicator variable). To test the assumption that AVF timing is a naturally ordered variable, a multinomial logit model was tested for model fit and predictive power (not shown). It produced predictive results no better than the ordered probit, so the ordered probit model was retained because of its intuitive appeal and simpler likelihood structure.

The selection-corrected cost model (table 3 ) shows that presence of comorbidity in most cases is associated with higher costs. All variables (including comorbid conditions and hospitalization days) were defined by the 12 months of experience before our study period to ensure that they were not confounded with cost outcomes for these groups. Surprisingly, the estimate of the age parameter is negative in each group, meaning that after accounting for other 
Table 3. Results of selection-corrected cost models on $\log (\cos t)$

\begin{tabular}{|c|c|c|c|c|c|c|}
\hline & \multicolumn{2}{|l|}{ mature } & \multicolumn{2}{|l|}{ maturing } & \multicolumn{2}{|c|}{ postinitiation } \\
\hline Lambda $^{1}$ & -0.0179 & 0.615 & 0.0268 & 0.268 & -0.0279 & 0.127 \\
\hline Age & -0.0049 & 0.001 & -0.0099 & 0.000 & -0.0056 & 0.000 \\
\hline Other race & 0.0025 & 0.950 & 0.0753 & 0.169 & -0.0572 & 0.053 \\
\hline ASHD & 0.0691 & 0.000 & 0.0333 & 0.140 & 0.0267 & 0.034 \\
\hline Congestive heart failure & 0.0225 & 0.315 & 0.0252 & 0.317 & -0.0134 & 0.333 \\
\hline Cerebrovascular accident & 0.0152 & 0.560 & 0.0008 & 0.978 & 0.0093 & 0.562 \\
\hline PVD & 0.0757 & 0.000 & -0.0049 & 0.836 & 0.0847 & 0.000 \\
\hline Other cardiac disease & -0.0193 & 0.397 & 0.0016 & 0.950 & 0.0209 & 0.147 \\
\hline Cancer & 0.0819 & 0.001 & 0.0359 & 0.225 & 0.0676 & 0.000 \\
\hline Diabetes & 0.0831 & 0.000 & 0.0439 & 0.038 & 0.0703 & 0.000 \\
\hline Wheelchair use & 0.1276 & 0.000 & 0.1030 & 0.000 & 0.1315 & 0.000 \\
\hline $\log$ (total hospital days) & 0.0139 & 0.410 & 0.0274 & 0.145 & 0.0128 & 0.210 \\
\hline At least 1 hospitalization & -0.0260 & 0.480 & 0.0084 & 0.845 & -0.0032 & 0.892 \\
\hline \multicolumn{7}{|c|}{ Geographic decile of average reimbursement ${ }^{2}$} \\
\hline 2 & 0.0326 & 0.489 & -0.0322 & 0.560 & -0.0169 & 0.606 \\
\hline 3 & 0.0479 & 0.324 & -0.0223 & 0.696 & 0.0438 & 0.186 \\
\hline 4 & 0.0439 & 0.343 & -0.0396 & 0.464 & 0.0145 & 0.645 \\
\hline 5 & 0.0672 & 0.155 & 0.0277 & 0.607 & 0.0679 & 0.035 \\
\hline 6 & 0.0953 & 0.039 & 0.0403 & 0.457 & 0.0838 & 0.008 \\
\hline
\end{tabular}

Reference categories are male sex, white race, absence of disease, geographic decile 1. ASHD = Atherosclerotic heart disease; $\mathrm{AVF}=$ arteriovenous fistula $\mathrm{COPD}=$ chronic obstructive pulmonary disease $\mathrm{GI}=$ gastrointestinal; $\mathrm{PVD}=$ peripheral vascular disease .

${ }^{1}$ Ordered probit variation of Heckman's Mills ratio. ${ }^{2}$ County-level deciles based on Dartmouth Atlas average reimbursement per Medicare beneficiary. ${ }^{3}$ Correlation between the ordered probit and $\log (\operatorname{cost})$ error terms. ${ }^{4}$ Standard deviation of the log(cost) error term.

factors, older age is associated with lower costs. This is likely related to earlier mortality, since older age is highly predictive of death and therefore cessation of dialysis expenses. Estimates of the age parameters within the cost model are relatively consistent across treatment groups, as are their estimated marginal effects, between USD 500 and USD 1,200 of savings for each additional year of age for each treatment group (data not shown).

The cost model produces an $\mathrm{R}^{2}$ value of $12.7 \%$. While low, this is consistent with $\mathrm{R}^{2}$ measures in the health claims literature, especially considering the difficulty of predicting claims for this select population.

We used the models (selection-corrected and cohortspecific ordinary least squares) to predict claims for each individual as if he or she were in each of the three treatment groups. These results were averaged across the actual treatment group and are displayed in figure 2. Compared with predictions from ordinary least squares models, the selection-corrected model shows a larger difference between the average predicted values for being in 


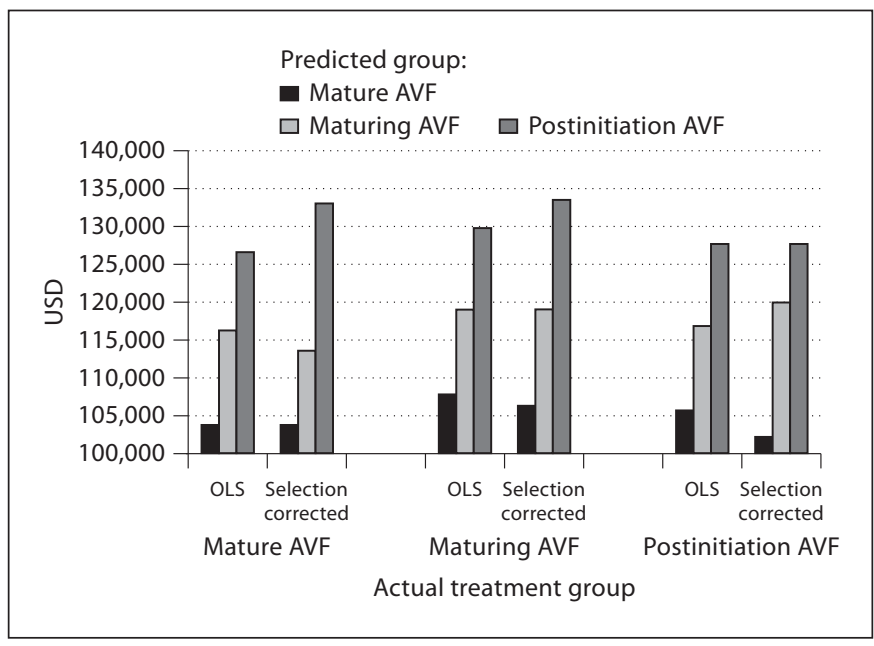

Fig. 2. Mean predicted cost from ordinary least squares vs. selection-corrected models. OLS = Ordinary least squares.

the mature AVF versus the postinitiation AVF group for each of the three cohorts, suggesting that statistical bias in the ordinary least squares models understates the cost savings in these data.

As noted, while actual and predicted costs for all patients are lowest in the mature AVF group, mortality is also lowest in that group, and lower mortality increases costs. To the extent that mortality differences are not identified by the selection adjustment in the inverse Mills ratio, estimates of cost savings associated with earlier AVF placement are likely to be conservative. During sensitivity testing, the entire process was repeated using only patients who survived for the first 12 months of dialysis, with similar results (not shown).

\section{Discussion}

In general, the results reflect much of what would be expected clinically. Patients who have a functioning AVF at dialysis initiation tend to be healthier and less frail than patients who initiate with a catheter. However, placing the AVF early enough to allow it to mature by the time of dialysis initiation also provides a substantial cost reduction over initiating dialysis with a still-maturing AVF. This observed cost difference is understated unless the estimation methods take into account the nonrandom treatment assignment. For example, predicting what would happen if a delayed AVF patient were moved to mature AVF status, to the extent that mortality differences are not identified by our health status measures, requires adjustment for nonrandom assignment to identify the savings due to the different access method without assuming the delayed AVF patient would live as long as the average (healthier) mature AVF patient. Ignoring these selection-related mortality differences understates the estimated savings.

The observed differences in costs between the groups likely result from many factors. Previously published studies have demonstrated that catheters are associated with higher rates of infections and complications $[5,7,12$, $13,18]$, possibly resulting in more frequent and longer hospitalizations. In our study this was certainly true; the percentages of patients in the mature AVF group who experienced a hospitalization for sepsis or vascular access infection after dialysis initiation were 4.8 and $3.4 \%$, respectively. Corresponding percentages for the maturing AVF group were 7.9 and $12.5 \%$, and for the postinitiation AVF group 20.5 and $14.6 \%$. In general, patients in the postinitiation AVF group were more often hospitalized for any reason (79.5\%) and stayed in the hospital longer [mean 3.46 days, standard error (SE) 0.04] than the maturing AVF (76.0\%; 3.18 days, SE 0.07) or mature AVF (64.0\%; 2.43 days, SE 0.05$)$ groups. Of note, these values are not adjusted for the nonrandom treatment assignment, but the direction of the association is consistent with published literature. Additionally, catheter use has been shown to be associated with higher doses of erythropoietin-stimulating agents and intravenous iron [20-22], which are also large cost drivers in the dialysis population.

Considering the lower rates of vascular access-related complications [7, 11-13, 18], and previous research demonstrating improved quality of life for AVF patients [34], it seems clear that patients who are good candidates for an AVF should have it placed early enough to assure maturation by the time of dialysis initiation.

Because patients with CKD may die before developing ESRD, it is necessary to consider how predialysis mortality could influence the cost savings of earlier AVF placement. Using the 2005 general Medicare 5\% random sample, non-ESRD, Medicare-eligible patients (aged $\geq 65$ years) with an AVF insertion can be identified and followed. We identified about 550 such patients; within 2 years of the AVF insertion claim, 71\% developed ESRD, $8 \%$ died without developing ESRD, and about $21 \%$ survived without developing ESRD. While isolating the exact dollar value that represents the cost of an AVF insertion is difficult, obtaining a rough estimate is possible using line-level revenue center information from claims. Data from claims indicate that a reasonable estimate of the cost to Medicare of an AVF insertion is USD 1,500-2,500. If 
$70 \%$ of CKD patients with an AVF develop ESRD, the cost per ESRD patient is between USD 2,100 and 3,500. Using a more conservative assumption of $50 \%$ of CKD patients developing ESRD, the cost per ESRD patient is between USD 3,000 and 5,000. Given that the average estimated total cost savings with a mature AVF are USD 12,801 for patients in the maturing AVF group (bootstrapped 95\% confidence interval 5,038-20,798) and USD 25,377 for patients in the postinitiation AVF group (5,973-39,267), preESRD mortality would not appear to negate the cost savings of initiating dialysis with a mature AVF.

Typically, about 110,000 patients (of all ages) initiate hemodialysis each year. According to the 2011 USRDS annual report (fig. 1.19ii) [35], about $15 \%$ of patients initiated using a catheter with a maturing AVF, and about $65 \%$ with a catheter alone; in our Medicare data, about $30 \%$ of patients who initiated with a catheter alone had an AVF placed later. Using these values, we estimated that of the 110,000 patients who initiate hemodialysis, about 16,500 do so with a maturing AVF and 21,450 with a catheter, but could have initiated with an AVF. We estimated the cost savings (net USD 5,000 AVF placement costs) for the 16,500 patients with maturing AVFs at USD 7,801; for the 21,450 patients with catheters who could have had AVFs, we estimated net savings at USD 20,377. This gave an estimated total net savings of USD 566 million (holding patient counts and AVF placement costs fixed, the bootstrapped confidence intervals imply a savings range of USD 21-996 million). Notably, these are conservative estimates since a larger proportion of younger catheter patients are likely to be good AFV candidates. Younger patients also experience greater benefit from early AVF placement [36] due to lower pre-ESRD mortality rates; including patients aged $<67$ years at initiation would demonstrate cost savings even greater than we demonstrate here. Regardless of whether Medicare extends coverage to this type of pre-ESRD treatment or health plans and large dialysis providers help cover this cost, the net savings appear to be significant.

Current barriers to AVF creation should be considered. Recent studies have cited procedural barriers (lack of formal policies for patient referral, etc.) and financial disincentives of predialysis care and inadequate reimbursement for surgeons $[37,38]$. These issues would likely need to be addressed to take full advantage of the cost savings associated with early AVF placement.

\section{Appropriateness of AVFs and Successful Maturation}

In recent years, AVFs have received considerable attention within the ESRD and dialysis communities. The advent of the Fistula First program and increased research surrounding AVFs correspond to an increase in AVF placement and use. However, some clinicians have noted that patient characteristics can often drive decisions relating to vascular access. For example, results from a recent survey [39] indicate that nephrologists would prefer that AVF patients be aged $<65$ years with minimal comorbidity and no history of a failed access. Different clinicians often come to different conclusions regarding the appropriateness of a surgical procedure, especially in a high-risk group. For this reason, we included only patients who eventually received an AVF in our analysis, trusting that this indicated a clinically appropriate AVF candidate.

Additionally, this study was performed on an intentto-treat basis, meaning that no consideration was given to what type of vascular access a patient actually used during any particular dialysis session. For example, if an AVF becomes clotted or infected, a patient may dialyze using a temporary catheter for one or two sessions. As this information is not available from the Medicare claims, making the distinction is currently impossible. However, from a policy standpoint, the intent-to-treat model is appropriate.

This study also did not consider the issue of successful maturation of AVFs, despite the fact that placement of an AVF does not necessarily mean it will mature to the point of use. A 2006 study [40] produced a risk equation for predicting whether a placed AVF would reach maturity without needing additional procedures or replacement. Four significant variables increased risk of AVF failure: age $\geq 65$ years, peripheral vascular disease, coronary artery disease, and non-white race. The authors went on to validate their equation, finding primary failure rates of placed AVFs ranging from $24 \%$ in the low-risk group to $69 \%$ in the high-risk group. Clearly, age and vascular disease influence the appropriateness of AVFs as well as their potential success. It was impossible from our data to accurately identify if or when an AVF failed.

Not all patients are ideal AVF candidates. Therefore, the question may be not whether to place an AVF sooner, but whether to place one at all. Some patients in our study cohort would likely be categorized by Lok's risk equation as at medium or even high risk for AVF failure (especially considering the cohort age), and later AVF placement may have been due to a clinician's determination of the proper course of action. The decision of whether and when to place an AVF should be made on an individual basis, and clinical recommendations should respect clinician autonomy. Our analyses assumed that because an 
AVF was ultimately placed, a clinician had determined that doing so was appropriate.

\section{Limitations}

While the data provide significant support for the validity of our IV, there is a possibility that it could be correlated with cost through non-AVF early monitoring and treatments. Though our descriptive statistics support use of 'NephDays' as an IV, lack of a formal test for its appropriateness is a limitation.

Patients aged $\geq 67$ years at the time of dialysis initiation are a subset of all incident dialysis patients. They are likely to have worse health and a higher disease burden than their younger counterparts. Event rates and overall costs might be very different, as age is a significant factor when clinicians determine the appropriateness of AVF placement. Therefore, results from this analysis are not directly generalizable to the entire population of incident dialysis patients. Informed by traditional mortality analyses, we would expect this older cohort to be more often of white race and female. However, while these patients may not represent all dialysis patients, they do represent a significant proportion of the hemodialysis population and its cost. According to the USRDS, of 101,306 incident hemodialysis patients in 2006, 51,182 (51\%) were aged $\geq 65$ years [41]. Additionally, of the USD 20.3 billion Medicare spent on all ESRD patients in 2006, 48\% (USD 9.8 billion) was accounted for by patients aged $\geq 65$ years.
This study also excluded patients due to health maintenance organization or payer status. Most excluded patients had Medicare Advantage coverage at some point during the period of interest. While they generated Medicare costs, full claims information was not available, making identification of those with AVFs impossible.

The age limitation in this study highlights the need for additional work. Future research could combine Medicare data with data from national insurance carriers to identify pre-Medicare costs, allowing a more refined understanding of how reduced complications from earlier AVF placement interact with improved mortality in a younger patient population.

\section{Conclusion}

Our results suggest that expanded coverage to include AVF placement prior to the current Medicare eligibility due to ESRD, timed so the AVF is expected to be mature at the time of dialysis initiation, may provide significant overall cost savings to Medicare, perhaps as much as USD 500 million per year. Additional studies, perhaps specifically targeted to a policy change regarding Medicare coverage of pre-ESRD care, are necessary. Continued study of dialysis vascular access is warranted to provide a full picture of how and when providers should place AVFs in patients who are candidates.

\section{Appendix: Diagnosis, Procedure, and Event Codes}

\begin{tabular}{ll}
\hline Comorbid condition & ICD-9-CM diagnosis codes \\
\hline Atherosclerotic heart disease & $410 . x-414 . x$, V45.81, V45.82 \\
Congestive heart failure & $398.91,422 . x, 425 . x, 428 . x, 402 . x 1,404 . x 1,404 . x 3, V 42.1$ \\
Cerebrovascular accident & $430 . x-438 . x$ \\
Peripheral vascular disease & $440 . x-444 . x, 447 . x, 451 . x-453 . x, 557 . x$ \\
Other cardiac disease & $420 . x, 421 . x, 423 . x, 424 . x, 429 . x, 785.0-785.3, \mathrm{~V} 42.2, \mathrm{~V} 43.3$ \\
Chronic obstructive pulmonary disease & $491 . x-494 . x, 496 . x, 510 . x$ \\
Gastrointestinal disease & $456.0-456.2,530.7,531 . x-534 . x, 569.84,569.85,578 . x$ \\
Liver disease & $570 . x, 571 . x, 572 . x, 572.4,573.1-573.3, V 42.7$ \\
Dysrhythmia & $426 . x, 427 . x, V 45.0, V 53.3$ \\
Cancer & $140 . x-172 . x, 174 . x-208 . x, 230 . x, 231 . x, 233 . x, 234 . x$ \\
Diabetes & $250 . x, 357.2,362.0,366.41$ \\
Anemia & $280 . x-285 . x$ \\
Chronic kidney disease & $016.0,095.4,189.0,189.9,223.0,236.91,250.4,271.4,274.1,283.11,403 . x 1,404 . x 2,404 . x 3$, \\
& $440.1,442.1,447.3,572.4,580 . x-588 . x, 591 . x, 642.1,646.2,753.12-753.17,753.19,753.2,794.4$ \\
Hypertension & $362.11,401 . x-405 . x, 437.2$ \\
AVF insertion & $C P T$ codes 36818, 36819, 36820, 36821, 36825 \\
\hline
\end{tabular}

AVF $=$ arteriovenous fistula CPT $=$ Current Procedural Terminology; ICD-9-CM = International Classification of Diseases, Ninth Revision, Clinical Modification. 


\section{Acknowledgments}

This study was performed as a deliverable under contract No. HHSN267200715002C (National Institute of Diabetes and Digestive and Kidney Diseases, National Institutes of Health, Bethesda, Md., USA). The authors thank USRDS colleagues Beth Forrest, Shane Nygaard, and Nan Booth, MSW, MPH, ELS, for regulatory assistance, assistance with manuscript preparation, and manuscript editing, respectively. We also thank the reviewers and editor for their helpful suggestions.

\section{Disclosure Statement}

The authors have no conflicts of interest with its subject matter.

\section{References}

1 Gilbertson DT, Liu J, Xue JL, Louis TA, Solid CA, Ebben JP, Collins AJ: Projecting the number of patients with end-stage renal disease in the United States to the year 2015. J Am Soc Nephrol 2005;16:3736-3741.

2 U.S. Renal Data System: USRDS 2010 Annual Data Report: Atlas of Chronic Kidney Disease and End-Stage Renal Disease in the United States, ed 2010, Bethesda, National Institutes of Health, National Institute of Diabetes and Digestive and Kidney Diseases, 2010.

-3 Spinowitz BS, Galler M, Golden RA, Rascoff JH, Schechter L, Held B, Charytan C: Subclavian vein stenosis as a complication of subclavian catheterization for hemodialysis. Arch Intern Med 1987;147:305-307.

$\checkmark 4$ Barrett N, Spencer S, McIvor J, Brown EA: Subclavian stenosis: a major complication of subclavian dialysis catheters. Nephrol Dial Transplant 1988;3:423-425.

5 National Kidney Foundation: An introduction to the kidneys and chronic kidney disease. 2011. http://www.kidney.org/patients/ plu/plu_intro/pluo_9.cfm (accessed February 2, 2012).

$\checkmark 6$ Astor BC, Eustace JA, Powe NR, Klag MJ, Fink NE, Coresh J: Type of vascular access and survival among incident hemodialysis patients: the Choices for Healthy Outcomes in Caring for ESRD (CHOICE) study. J Am Soc Nephrol 2005;16:1449-1455.

7 Perera GB, Mueller MP, Kubaska SM, Wilson SE, Lawrence PF, Fujitani RM: Superiority of autogenous arteriovenous hemodialysis access: maintenance of function with fewer secondary interventions. Ann Vasc Surg 2004;18:66-73.

8 Mehta S: Statistical summary of clinical results of vascular access procedures for hemodialysis; in Sommer BG, Henry ML (eds): Vascular Access for Hemodialysis - II. Chicago, W. L. Gore \& Associates, 1991, pp 145 157.

9 Eggers P, Milam R: Trends in vascular access procedures and expenditures in Medicare's ESRD program; Vascular Access for Hemodialysis - VII. Chicago, Gore, 2001, pp 133 143.
10 Polkinghorne KR, McDonald SP, Atkins RC, Kerr PG: Vascular access and all-cause mortality: a propensity score analysis. J Am Soc Nephrol 2004;15:477-486.

11 Feldman HI, Held PJ, Hutchinson JT, Stoiber E, Hartigan MF, Berlin JA: Hemodialysis vascular access morbidity in the United States. Kidney Int 1993;43:1091-1096.

12 Nassar GM, Ayus JC: Infectious complications of the hemodialysis access. Kidney Int 2001;60:1-13.

13 Gulati S, Sahu KM, Avula S, Sharma RK, Ayyagiri A, Pandey CM: Role of vascular access as a risk factor for infections in hemodialysis. Ren Fail 2003;25:967-973.

- 14 Dhingra RK, Young EW, Hulbert-Shearon TE, Leavey SF, Port FK: Type of vascular access and mortality in U.S. hemodialysis patients. Kidney Int 2001;60:1443-1451.

15 Xue JL, Dahl D, Ebben JP, Collins AJ: The association of initial hemodialysis access type with mortality outcomes in elderly Medicare ESRD patients. Am J Kidney Dis 2003;42: 1013-1019.

16 Schlieper G, Kruger T, Djuric Z, Damjanovic T, Markovic N, Schurgers LJ, Brandenburg VM, Westenfeld R, Dimkovic S, Ketteler M, Grootendorst DC, Dekker FW, Floege J, Dimkovic N: Vascular access calcification predicts mortality in hemodialysis patients. Kidney Int 2008;74:1582-1587.

17 Woods JD, Port FK: The impact of vascular access for haemodialysis on patient morbidity and mortality. Nephrol Dial Transplant 1997; 12:657-659.

18 Ishani A, Collins AJ, Herzog CA, Foley RN: Septicemia, access and cardiovascular disease in dialysis patients: the USRDS Wave 2 study. Kidney Int 2005;68:311-318.

19 Raithatha A, McKane W, Kendray D, Evans C: Catheter access for hemodialysis defines higher mortality in late-presenting dialysis patients. Ren Fail 2010;32:1183-1188.

20 Lopez-Gomez JM, Portoles JM, Aljama P: Factors that condition the response to erythropoietin in patients on hemodialysis and their relation to mortality. Kidney Int 2008; 74(suppl 111):S75-S81.
21 Chand DH, Teo BW, Fatica RA, Brier M: Influence of vascular access type on outcome measures in patients on maintenance hemodialysis. Nephron Clin Pract 2008;108:c91c98.

22 Goicoechea M, Caramelo C, Rodriguez P, Verde E, Gruss E, Albalate M, Ortiz A, Casado S, Valderrabano F: Role of type of vascular access in erythropoietin and intravenous iron requirements in haemodialysis. Nephrol Dial Transplant 2001;16:2188-2193.

23 National Kidney Foundation: NKF K/DOQI guidelines: clinical practice guidelines and clinical practice recommendations, 2006 updates hemodialysis adequacy, peritoneal dialysis adequacy, vascular access. 2006. http:// www.kidney.org/PROFESSIONALS/kdoqi/ guideline_upHD_PD_VA/va_guide1.htm (accessed February 2, 2012).

24 Albers FJ: Causes of hemodialysis access failure. Adv Ren Replace Ther 1994;1:107-118.

25 Schon D, Blume SW, Niebauer K, Hollenbeak CS, de LG: Increasing the use of arteriovenous fistula in hemodialysis: economic benefits and economic barriers. Clin J Am Soc Nephrol 2007;2:268-276.

-26 Wu LC, Lin MY, Hsieh CC, Chiu HC, Mau LW, Chiu YW, Chen HC, Hwang SJ: Planned creation of vascular access saves medical expenses for incident dialysis patients. Kaohsiung J Med Sci 2009;25:521-529.

27 The Cost Effectiveness of Alternative Types of Vascular Access and the Economic Cost of ESRD. Bethesda, National Institutes of Health, National Institute of Diabetes and Digestive and Kidney Disorders, 1995, pp 139-157.

28 Foley RN, Murray AM, Li S, Herzog CA, McBean AM, Eggers PW, Collins AJ: Chronic kidney disease and the risk for cardiovascular disease, renal replacement, and death in the United States Medicare population, 1998 to 1999. J Am Soc Nephrol 2005; 16:489-495.

29 Heckman JJ: Sample selection bias as a specification error. Econometrica 1979;47:153161. 
- 30 Chiburis R, Lokshin M: Maximum likelihood and two-step estimation of an orderedprobit selection model. Stata J 2007;7:167182.

-31 Manning WG: The logged dependent variable, heteroscedasticity, and the retransformation problem. J Health Econ 1998; 17:283295.

32 Manning WG, Mullahy J: Estimating log models: to transform or not to transform? J Health Econ 2001;20:461-494.

33 Duan N: Smearing estimate: a nonparametric retransformation method. J Am Stat Assoc 1983;383:605-610.

34 Wasse H, Kutner N, Zhang R, Huang Y: Association of initial hemodialysis vascular access with patient-reported health status and quality of life. Clin J Am Soc Nephrol 2007; 2:708-714.
35 U.S. Renal Data System: USRDS 2011 Annual Data Report: Atlas of Chronic Kidney Disease and End-Stage Renal Disease in the United States. ed 2011. Bethesda, National Institutes of Health, National Institute of Diabetes and Digestive and Kidney Diseases, 2011.

36 O’Hare AM, Bertenthal D, Walter LC, Garg AX, Covinsky K, Kaufman JS, Rodriguez RA, Allon M: When to refer patients with chronic kidney disease for vascular access surgery: should age be a consideration? Kidney Int 2007;71:555-561.

37 Lopez-Vargas PA, Craig JC, Gallagher MP, Walker RG, Snelling PL, Pedagogos E, Gray NA, Divi MD, Gillies AH, Suranyi MG, Thein H, McDonald SP, Russell C, Polkinghorne KR: Barriers to timely arteriovenous creation: a study of providers and patients. Am J Kidney Dis 2011;57:873-882.

38 Schon D, Blume SW, Niebauer K, Hollenbeak CS, de Lissovoy G: Increasing the use of arteriovenous fistula in hemodialysis: economic benefits and economic barriers. Clin J Am Soc Nephrol 2007;2:268-276.
39 Xi W, MacNab J, Lok CE, Lee TC, Maya ID, Mokrzycki MH, Moist LM: Who should be referred for a fistula? A survey of nephrologists. Nephrol Dial Transplant 2010;25: 2644-2651.

40 Lok CE, Allon M, Moist L, Oliver MJ, Shah $\mathrm{H}$, Zimmerman D: Risk equation determining unsuccessful cannulation events and failure to maturation in arteriovenous fistulas (REDUCE FTM I). J Am Soc Nephrol 2006; 17:3204-3212.

41 U.S. Renal Data System: USRDS 2008 Annual Data Report: Atlas of Chronic Kidney Disease and End-Stage Renal Disease in the United States. Bethesda, National Institutes of Health, National Institute of Diabetes and Digestive and Kidney Diseases, 2008. 